\title{
ULTRASONIC ATTENUATION IN DIELECTRIC CRYSTALS
}

\author{
S.K. KOR AND R.K. SINGH \\ Department of Physics, University of Allahabad \\ Allahabad - 211002 , India \\ (Received August 27, 1991)
}

\begin{abstract}
Ultrasonic attenuation and non-linearity parameters have been evaluated for dielectric crystals $\mathrm{RbCl}, \mathrm{RbBr}$ and $\mathrm{RbI}$ in a wide temperature range. Basic physical parameters, nearest neighbour distance and hardness parameters of the substance and Coulomb and Born-Mayer potentials have been used to obtain ultrasonic attenuation due to phonon-viscosity mechanism along $\langle 100\rangle$ direction of propagation for longitudinal and shear waves. Results are as expected and it has been concluded that ultrasonic attenuation is a fundamental property of the substance.
\end{abstract}

PACS numbers: 62.20.Dc, 62.65.+K

\section{Introduction}

Ultrasonic attenuation techniques have been used as a versatile tool for the determination of internal structure and other inherent properties of solids [1-5]. Ultrasonic attenuation studies have been made for metallic $[6,7]$, semiconducting $[8,9]$ and dielectric $[10]$ substances. Of the three dominant interactions responsible for ultrasonic attenuation in solids, electron-lattice interaction is principal cause of acoustical dissipation in metals [8], at low temperatures, while phonon-phonon interaction is the main cause of acoustical attenuation in all types of solids at higher temperatures, provided the substance is perfect, non-ferromagnetic and non-ferroelectric as established by other workers $[8,11]$. The prominent mechanism for ultrasonic attenuation in solids is the Akhiezer mechanism [12], in which the attenuation results because, during sound wave propagation through solids, equilibrium distribution of thermal phonons is disturbed, and reestablishment of equilibrium is a relaxational phenomenon, in which entropy is produced and energy is lost from the sound wave.

In the present communication, we have studied ultrasonic attenuation due to phonon-phonon interaction in rubidium halide crystals viz. rubidium chloride, 
rubidium bromide and rubidium iodide at different temperatures viz. 100, 200, 300 and $400 \mathrm{~K}$, using nearest neighbour distance, hardness parameter, Coulomb and Born-Mayer potentials and following Mason's approach [8]. The average Grüneisen parameters, nonlinearity parameters and attenuation coefficients for longitudinal and shear waves are evaluated along $\langle 100\rangle$ direction of propagation.

\section{Theory}

The theory consists of two parts. In the first part a temperature variation of second and third order elastic constants has been discussed, while in the next part ultrasonic attenuation due to phonon-phonon interaction at different temperatures is to be considered.

\subsection{Theory of elastic constants}

Assuming Coulomb or electrostatic potential $\left( \pm e^{2} / r\right)$ and Born-Mayer [13] type potential $Q(r)=A \exp (-r / \rho), e, r$, and $\rho$ being electronic charge, nearest neighbour distance and hardness parameter, and following Brugger's [14] definition of elastic constants at absolute zero, and taking interactions up to second nearest neighbours, the second and third order elastic constants (SOEC $C_{i j}^{0}$ and TOEC $C_{i j k}^{0}$ in Voigt notation) are obtained as shown in Table I. Various lattice sums are shown in Table II.

TABLE I

SOEC and TOEC at absolute zero.

\begin{aligned} \hline \hline$C_{11}^{0}= & (3 / 2)\left(e^{2} / r_{0}^{4}\right) S_{5}^{2}+\left(1 / \rho r_{0}\right)\left(1 / r_{0}+1 / \rho\right) Q\left(r_{0}\right) \\ & +\left(2 / \rho r_{0}\right)\left(\sqrt{2} / 2 r_{0}+1 / \rho\right) Q\left(\sqrt{2} r_{0}\right) \\ C_{12}^{0}= & C_{44}^{0}=(3 / 2)\left(e^{2} / r_{0}^{4}\right) S_{5}^{1,1}+\left(1 / \rho r_{0}\right)\left(\sqrt{2} / 2 r_{0}+1 / \rho\right) Q\left(\sqrt{2} r_{0}\right) \\ C_{111}^{0}= & (15 / 2)\left(e^{2} / r_{0}^{4}\right) S_{7}^{3}-(1 / \rho)\left(3 / r_{0}^{2}+3 / \rho r_{0}+1 / \rho^{2}\right) Q\left(r_{0}\right) \\ & -(1 / 2 \rho)\left(3 \sqrt{2} / r_{0}^{2}+6 / \rho r_{0}+2 \sqrt{2} / \rho^{2}\right) Q\left(\sqrt{2} r_{0}\right) \\ C_{112}^{0}= & C_{166}^{0}=-(15 / 2)\left(e^{2} / r_{0}^{4}\right) S_{7}^{2,1}-(1 / 4 \rho)\left(3 \sqrt{2} / r_{0}^{2}+6 / \rho r_{0}+2 \sqrt{2} / \rho^{2}\right) Q\left(\sqrt{2} r_{0}\right) \\ C_{123}^{0}= & C_{144}^{0}=C_{456}^{0}=-(15 / 2)\left(e^{2} / r_{0}^{4}\right) S_{7}^{1,1,1}\end{aligned}$

TABLE II

\begin{tabular}{l|l|}
\multicolumn{2}{c}{ Various lattice sums. } \\
\hline \hline$S_{3}^{1}=-0.58252$ & $S_{5}^{2}=-1.04622$ \\
$S_{5}^{1,1}=0.23185$ & $S_{7}^{3}=-1.36852$ \\
$S_{7}^{2,1}=0.16115$ & $S_{7}^{1,1,1}=-0.09045$
\end{tabular}

According to the anharmonic theory of lattice dynamics developed by Leibfried and Ludwig [15] or Hahn et al. [16], the change in elastic constant occurs due to change in vibrational energy of the crystal. Thus adding vibrational energy contributions we get SOEC and TOEC at required temperature as:

$$
C_{i j}(T)=C_{i j}^{0}+C_{i j}^{\text {vib. }} \text { and } C_{i j k}(T)=C_{i j k}^{0}+C_{i j k}^{\text {vib. }} .
$$

This theory $[17,18]$ has been used earlier to obtain elastic constants. The vibrational energy contributions are presented in Table III. Various $f^{n}$ 's and $G_{n}$ 's are given in Table IV and Table V. 


\subsection{Theory of phonon-viscosity mechanism}

SOEC's and TOEC's thus obtained are used to obtain Grüneisen parameters $\left\langle\gamma_{i}^{j}\right\rangle$ and $\left\langle\gamma_{i}^{j 2}\right\rangle$, using Grüneisen tables [8] along $\langle 100\rangle$ direction of propagation for longitudinal and shear waves. These Grüneisen parameters are related with Mason's nonlinearity constant as:

$$
D=9\left\langle\gamma_{i}^{j 2}\right\rangle-3 C_{v} T\left\langle\gamma_{i}^{j}\right\rangle^{2} / E_{0},
$$

where $C_{v}$ is specific heat per unit volume, $T$ absolute temperature, $E_{0}$ is the energy of the crystal, $\left\langle\gamma_{i}^{j}\right\rangle$ and $\left\langle\gamma_{i}^{j 2}\right\rangle$ are average Grüneisen number and square average Grüneisen numbers over 39 pure modes for longitudinal wave and over 18 pure modes for shear waves.

$$
\begin{aligned}
& \text { Vibrational energy contributions. TABLE III } \\
& \hline \hline C_{11}^{\text {vib. }}=f^{1,1} G_{1}^{2}+f^{2} G_{2} \\
& C_{12}^{\text {vib. }}=f^{1,1} G_{1}^{2}+f^{2} G_{1,1} \\
& C_{44}^{\text {vib. }}=f^{2} G_{1,1} \\
& C_{111}^{\text {vib. }}=f^{1,1,1} G_{1}^{3}+3 f^{2,1} G_{2} G_{1}+f^{3} G_{3} \\
& C_{112}^{\text {vib. }}=f^{1,1,1} G_{1}^{3}+f^{2,1}\left(2 G_{1,1}+G_{2}\right) G_{1}+f^{3} G_{2,1} \\
& C_{123}^{\text {vib. }}=f^{1,1,1} G_{1}^{3}+3 f^{2,1} G_{1} G_{1,1}+f^{2} G_{1,1,1} \\
& C_{144}^{\text {vib. }}=f^{2,1} G_{1} G_{1,1}+f^{3} G_{1,1,1} \\
& C_{456}^{\text {vib }}=f^{3} G_{1,1,1} \\
& C_{166}^{\text {vib }}=f^{2,1} G_{1} G_{1,1}+f^{3} G_{2,1}
\end{aligned}
$$

Expressions for various $f^{n}$ 's. TABLE IV

\begin{tabular}{l}
\hline \hline$f^{2}=f^{3}=\left(1 / 2 r_{0}^{3}\right)\left(\hbar w_{0} / 4\right) \operatorname{coth} X$ \\
$f^{1,1}=f^{2,1}=-\left(1 / 2 r_{0}^{3}\right)\left(\hbar w_{0} / 48\right)\left(X / \sinh ^{2} X+\operatorname{coth} X\right)$ \\
$f^{1,1,1}=\left(1 / 2 r_{0}^{3}\right)\left(\hbar w_{0} / 192\right)\left(\left(\hbar w_{0} / k T\right)^{2}\left(\operatorname{coth} X / 6 \sinh ^{2} X\right)+X / \sinh ^{2} X+\operatorname{coth} X\right)$ \\
$X \quad=\hbar w_{0} / 2 k T, \hbar=h / 2 \pi, k$ is the Boltzmann constant, \\
$w_{0}^{2}=\left(1 / M_{+}+1 / M_{-}\right)\left(1 / H_{\rho} r_{0}\right)$ \\
$M_{+}$and $M_{-}$are masses of positive and negative ions
\end{tabular}

$$
\begin{array}{ll}
\text { Expressions for various } G_{n} \text { 's. } & \text { TABLE V } \\
\hline \hline G_{1}=2\left[\left(2+\rho_{0}-\rho_{0}^{2}\right) Q\left(r_{0}\right)+2\left(\sqrt{2}+2 \rho_{0}-\sqrt{2} \rho_{0}^{2}\right) Q\left(\sqrt{2} r_{0}\right)\right] H \\
G_{2}=2\left[\left(-6-6 \rho_{0}-\rho_{0}^{2}+\rho_{0}^{3}\right) Q\left(r_{0}\right)\right] H+2 G_{1,1} \\
G_{3}=2\left[\left(30+30 \rho_{0}+9 \rho_{0}^{2}-\rho_{0}^{3}-\rho_{0}^{4}\right) Q\left(r_{0}\right)\right] H+2 G_{2,1} \\
G_{1,1}=\left(-3 \sqrt{2}-6 \rho_{0}-\sqrt{2} \rho_{0}^{2}+2 \rho_{0}^{3}\right) Q\left(\sqrt{2} r_{0}\right) H \\
G_{2,1}=\left[(15 / 2) \sqrt{2}+15 \rho_{0}+(9 / 2) \sqrt{2} \rho_{0}^{2}-\rho_{0}^{3}-\sqrt{2} \rho_{0}^{4}\right] Q\left(\sqrt{2} r_{0}\right) H \\
G_{1,1,1}=0 \\
H \quad=\left[\left(\rho_{0}-2\right) Q\left(r_{0}\right)+2\left(\rho_{0}-\sqrt{2}\right) Q\left(\sqrt{2} r_{0}\right)\right]^{-1} \\
\rho_{0} \quad=r_{0} / \rho \\
A \quad=-3 S_{3}^{1}\left(e^{2} / r_{0}^{2}\right)\left[6 \exp \left(-\rho_{0}\right)+12 \sqrt{2} \exp \left(-\rho_{0} \sqrt{2}\right)\right]^{-1} \\
Q\left(r_{0}\right)=A \exp \left(-r_{0} / \rho\right) \text { and } Q\left(\sqrt{2} r_{0}\right)=A \exp \left(-\sqrt{2} r_{0} / \rho\right)
\end{array}
$$

Using $\left\langle\gamma_{i}^{j}\right\rangle,\left\langle\gamma_{i}^{j 2}\right\rangle$ and nonlinearity constants following relations for phonon-viscosity loss are obtained [8] for ultrasonic frequencies in the range $\omega \tau_{\text {th }} \ll 1$ :

$$
\left(\alpha / f^{2}\right)_{p-p}=(2 \pi)^{2} E_{0}(D / 3) \tau_{\mathrm{th}} / d v^{3},
$$


where $d$ is density, $\tau_{\text {th }}$ is thermal relaxation time for the exchange of acoustic and thermal energies, given as

$$
(1 / 2) \tau_{1}=\tau_{\mathrm{s}}=\tau_{\text {th }}=3 K / C_{v} \bar{v}^{2},
$$

where $v$ represents $v_{1}$ (longitudinal wave velocity) or $v_{\mathbf{s}}$ (shear wave velocity). These are related with $C_{i j}$ 's as:

$$
v_{1}=\left(C_{11} / d\right)^{1 / 2} \text { and } v_{\mathrm{s}}=\left(C_{44} / d\right)^{1 / 2} \text { and } 3 / \bar{v}^{3}=\left(1 / v_{1}^{3}+2 / v_{\mathrm{s}}^{3}\right) \text {, }
$$

where $\bar{v}$ is the Debye average velocity.

\section{Evaluation}

The constants $C_{i j}$ and $C_{i j k}$ at different temperatures are evaluated using Born-repulsive parameter $(\mathrm{RbCl}=0.283, \mathrm{RbBr}=0.293, \mathrm{RbI}=0.298)$ all in $\mathrm{A}^{0}$ and nearest neighbour distance [19]. Using $C_{i j}$ and $C_{i j k}$ thus obtained, average Grüneisen numbers are computed along $\langle 100\rangle$ direction for longitudinal and transverse waves using Mason's tables [8]. The thermal relaxation time $\tau_{\text {th }}$ is obtained using thermal conductivity [20] and specific heat [21] with the help of equations (4) and (5). Nonlinearity coupling constant $D$ is obtained using specific heat and energy of the crystal [21] as a function of temperature and is shown in Table VI. Phonon-viscosity loss at different temperatures is obtained and is shown in Table VII and Fig. 1.

\section{TABLE VI}

$D_{\mathrm{l}}, D_{\mathrm{s}}$ and $D_{\mathrm{l}} / D_{\mathrm{s}}$ at different temperatures in rubidium halides along $\langle 100\rangle$ directions.

\begin{tabular}{l|c|r|r|r|r}
\hline \hline Substance & $D$ & $100 \mathrm{~K}$ & $200 \mathrm{~K}$ & $300 \mathrm{~K}$ & $400 \mathrm{~K}$ \\
\hline \multirow{3}{*}{$\mathrm{RbCl}$} & $D_{\mathrm{l}}$ & 43.56 & 42.88 & 40.63 & 38.78 \\
& $D_{\mathrm{s}}$ & 2.97 & 2.94 & 2.90 & 2.87 \\
& $D_{\mathrm{l}} / D_{\mathrm{s}}$ & 14.62 & 14.57 & 13.97 & 13.49 \\
\hline \multirow{3}{*}{$\mathrm{RbBr}$} & $D_{\mathrm{l}}$ & 43.80 & 42.21 & 40.07 & 38.12 \\
& $D_{\mathrm{s}}$ & 2.98 & 2.94 & 2.91 & 2.87 \\
& $D_{\mathrm{l}} / D_{\mathrm{s}}$ & 14.64 & 14.31 & 13.76 & 13.26 \\
\hline \multirow{3}{*}{$\mathrm{RbI}$} & $D_{\mathrm{l}}$ & 50.85 & 48.46 & 45.90 & 43.73 \\
& $D_{\mathrm{s}}$ & 2.91 & 2.88 & 2.84 & 2.82 \\
& $D_{\mathrm{l}} / D_{\mathrm{s}}$ & 17.45 & 16.82 & 16.11 & 15.50
\end{tabular}

TABLE VII

Ultrasonic attenuation for longitudinal $\left(\alpha / f^{2}\right)_{1}$ and shear wave $\left(\alpha / f^{2}\right)_{\mathrm{s}}$ in $10^{-18} \mathrm{~Np} \mathrm{~s}^{2} \mathrm{~cm}^{-1}$.

\begin{tabular}{l|r|r|r|r|r|r}
\hline \hline \multirow{2}{*}{ Temp. K } & \multicolumn{2}{|c|}{$\mathrm{RbCl}$} & \multicolumn{2}{c|}{$\mathrm{RbBr}$} & \multicolumn{2}{c}{$\mathrm{RbI}$} \\
\cline { 2 - 7 } & $\left(\alpha / f^{2}\right)_{l}$ & $\left(\alpha / f^{2}\right)_{\mathrm{s}}$ & $\left(\alpha / f^{2}\right)_{1}$ & $\left(\alpha / f^{2}\right)_{\mathrm{s}}$ & $\left(\alpha / f^{2}\right)_{1}$ & $\left(\alpha / f^{2}\right)_{\mathrm{s}}$ \\
\hline 100 & 4.46 & 2.09 & 19.60 & 8.68 & 48.90 & 23.60 \\
200 & 9.07 & 4.00 & 27.32 & 13.51 & 65.81 & 35.10 \\
300 & 11.62 & 6.32 & 32.10 & 17.50 & 76.30 & 45.40 \\
400 & 14.24 & 8.45 & 36.45 & 21.76 & 89.20 & 58.60
\end{tabular}




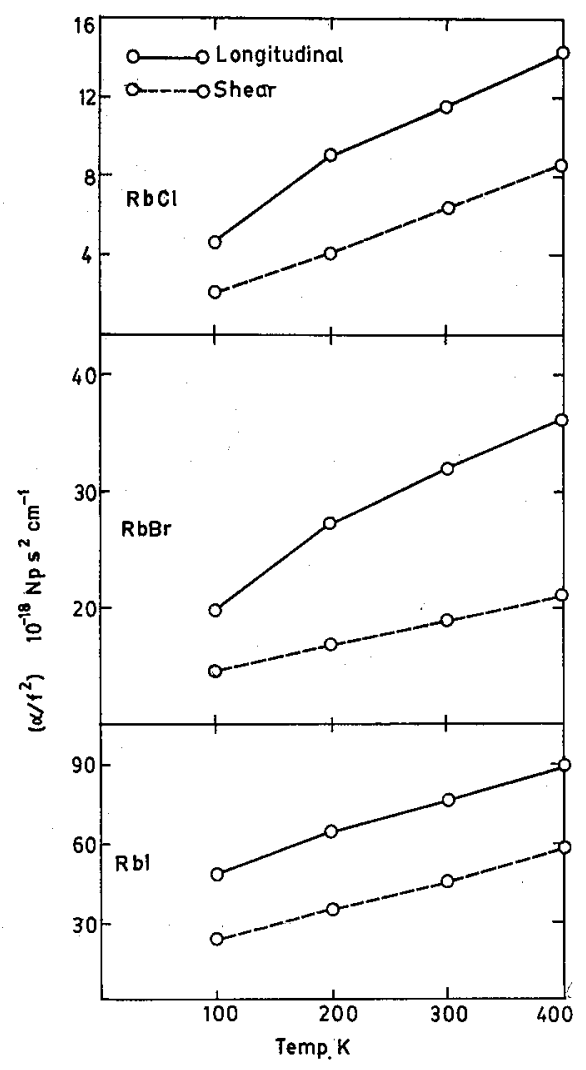

Fig. 1. Ultrasonic attenuation values for rubidium halides along $\langle 100\rangle$ direction for longitudinal and shear waves at different temperatures.

\section{Results and conclusions}

The best check for the validity of the present investigation will be to compare nonlinearity coupling constants $(D)$ and phonon-viscosity loss $\left(\alpha / f^{2}\right)_{p-p}$ with other similar solids.

\subsection{Nonlinearity coupling constant}

The values of nonlinearity parameters for the three dielectric crystals are shown in Table VI. The nonlinearity parameters ratio $\left(D_{\mathrm{l}} / D_{\mathrm{s}}\right)$ lies between 14 to 17 , which is as expected [22,23]. Values of $D_{1}$ and $D_{\mathrm{s}}$ are found to increase from $\mathrm{RbCl}$ to $\mathrm{RbI}$. Also, $D_{1}$ and $D_{\mathrm{s}}$ are decreasing with temperature along $\langle 100\rangle$ direction and $D$ is larger for longitudinal wave than the shear wave like other alkali halide crystals [22]. 


\subsection{Phonon-viscosity loss}

Temperature dependence of the ultrasonic attenuation due to phonon-phonon interaction for longitudinal and shear waves is shown in Table VII and Fig. 1. At $100 \mathrm{~K}$ magnitude of attenuation is the smallest, and as we go towards higher temperatures it increases and follows $T^{n}$ law, where $n$ lies between 0.40 and 0.55 for both longitudinal and shear waves. It is found that attenuation for shear wave is less than the longitudinal wave along $\langle 100\rangle$ direction of propagation, which is observed in other alkali halide crystals [22]. It has also been found that attenuation increases as molecular weight increases (from $\mathrm{RbCl}$ to $\mathrm{RbI}$ ).

As the whole evaluation is done starting from the basic physical parameters of the substance and using electrostatic and Born-Mayer type potentials, it suffices to conclude that ultrasonic attenuation is a fundamental property of the substance.

\section{References}

[1] P. Schrey, J. Phys. (France) 42, C5-671 (1981).

[2] J.P. Bonnet, M. Boisser, C. Vedel, R. Vacher, J. Phys. Chem. Solids 44, 515 (1983).

[3] J. Krautkramer, H. Krautkramer, Ultrasonic Study of Materials, 3rd ed. Academic Press, New York 1983.

[4] M.S. Anderson, C.A. Swenson, Phys. Rev. B, Condens. Matter 31, 668 (1985).

[5] T. Soma, H.M. Kagaya, Phys. Status Solidi B 126, 91 (1984).

[6] S.K. Kor, R.P. Khare, Acta Phys. Pol. A58, 725 (1980).

[7] S.K. Kor; Kailash, K. Shanker, P. Mehrotra, J. Phys. Soc. Jpn. 7, 2428 (1987).

[8] W.P. Mason, Physical Acoustics, Academic Press, New York 3B,1965.

[9] S.S. Shukla, S.S. Yun, J. Acoust. Soc. Am. 70, 1713 (1981).

[10] S.K. Kor, Kailash, Acta Phys. Pol. A71, 9 (1982).

[11] S.K. Kor, R.R. Yadav, Kailash, J. Phys. Soc. Jpn. 55, 207 (1986).

[12] A. Akkheser, J. Phys. (USSR) 1, 227 (1939).

[13] M. Born, J. Mayer, Z. Phys. 75, 1 (1932).

[14] K. Brugger, Phys. Rev. A 133, 1611 (1964).

[15] G. Leibfried, W. Ludwig, Solid State Physics, Vol. 12, Academic Press, New York 1961.

[16] G. Leibfried, H. Hahn, Z. Phys. 150, 150 (1958).

[17] P.B. Ghate, Phys. Rev. A 139, 1966 (1965).

[18] S. Mori, Y. Hiki, J. Phys. Soc. Jpn. 45, 1449 (1978).

[19] M.P. Tosi, Solid State Physics, Vol. 16, Academic Press, New York 1965.

[20] CRC Handbook of Chemistry and Physics, 58th ed., CRS Press, Florida, 1977-1978.

[21] American Institute of Physics Handbook, 3rd ed., Academic Press, New York 1963.

[22] Kailash, Ph. D. Thesis, Allahabad University, India, 1986.

[23] W.P Mason, R. Rosenberg, J. Acoust. Soc. Am. 95, 47 (1969). 